\title{
EL PEGADO ELÁSTICO EN FACHADAS TRASVENTILADAS DE PIEDRA
}

\author{
Antonio Raya de Blas, Cristóbal Crespo González, \\ Santiago Sánchez Iglesias y Enrique Antelo Tudela
}

The elastic bonding in stone rain-screen

Boletín Académico. Revista de investigación y arquitectura contemporánea Escuela Técnica Superior de Arquitectura. Universidade da Coruña elSSN 2173-6723

unw.boletinacademico.com

Número 2 (2012)

Páginas 36-45

Fecha de recepción 31.10.2011

Fecha de aceptación 02.02.2012

https://doi.org/10.17979/bac.2012.2.0.975

\begin{abstract}
Resumen
Partiendo de un posicionamiento arquitectónico, este artículo propone la resolución técnica de los requerimientos formales de una fachada trasventilada en un proyecto concreto, y describe la investigación realizada para su comprobación y construcción. La propuesta concreta es contrastada posteriormente en otros edificios, desarrollándose una solución constructiva alternativa al anclaje mecánico de las placas de piedra.
\end{abstract}

\section{Abstract}

This article, based on an architectural position, proposes a technical solution of the formal requirements of a rain screen wall on a specific project, and describes the process of research made for its verification and construction. The solution was subsequently proved in other buildings in order to develop an alternative solution to stone mechanical anchoring.

\section{Palabras clave}

Pegado, elástico, fachada, trasventilada, piedra.

\section{Keywords}

Bonding, elastic, facade, rain-screen, stone. 


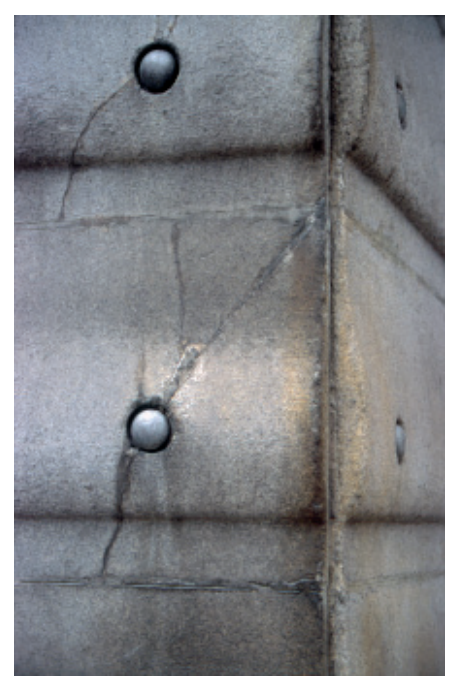

Wall-spacer

Garden Hill, Hartfield, Sussex

This object, from the bath-house of a Roman villa, formed part of a system which separated the two skins of the wall by means of iron rods clad with fired-clay reel-shaped spacers. PRBPASA5:12

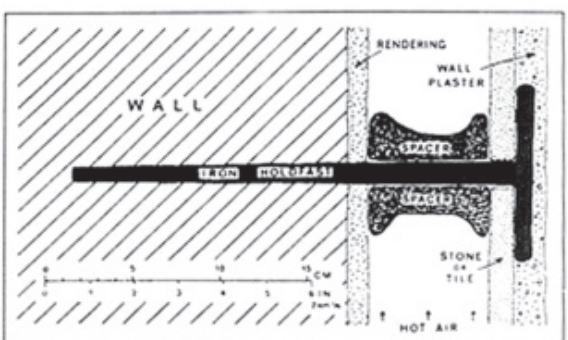

Reconstruction of use of baked clay 'spacer' and iron hold-fast. After J.H. Money

1 (Izda.) Otto Wagner, Postsparkasse (Caja Postal), Viena (1904/06); detalle donde se aprecia el uso de aplacado de piedra con anclajes vistos. 2 (Dcha.) "Wall-spacer" del British Museum, según J.H. Money; reconstrucción del detalle de doble piel con cámara de circulación de aire caliente. Un clavo de hierro con un separador del mismo material, ancla a un muro revestido una hoja de piedra o azulejo con un acabado de yeso que oculta la cabeza del anclaje.

\section{INTRODUCCIÓN}

La investigación de soluciones constructivas que resuelvan problemas formales, técnicos y económicos planteados en la arquitectura, tal vez sea uno de los campos de investigación más frecuentados por los arquitectos, a través de la metodología inherente a una actividad profesional en la que los aspectos compositivos juegan un papel determinante. Todos tenemos en la memoria ejemplos como el de Postsparkasse o Karlplatz (Fig. 01), donde Otto Wagner desarrolla las teorías de su libro sobre los aplacados delgados de piedra ${ }^{1}$.

Pero volvamos al proceso de investigación al que hacíamos referencia, donde se parte de una exigencia formal generada por la creación arquitectónica, y se investiga una materialización constructiva que aplique, reinterprete o genere una solución técnica posible. Se contrasta ésta solución con la idea arquitectónica y se comprueba su viabilidad económica, en un proceso cuajado de constantes interacciones no lineales.

Bien es cierto que la necesidad expresiva de la obra arquitectónica nos acerca a metodologías de investigación más propias de otras artes, como la pintura o la escultura, pero la carga técnica y la trascendencia económica de las soluciones constructivas nos alejan radicalmente de las mismas, sin acercarnos a los métodos científicos puros que no consideran los condicionantes formales y compositivos. Existen estudios sobre creaciones arquitectónicas cercanos al concepto de ciencia normal (concepto de paradigma) de Thomas $\mathrm{Kuhn}^{2}$, pero de escaso interés para los procesos de creación de los arquitectos con sus necesidades inherentes de innovación formal y técnica.

Siguiendo este discurso, aquí se describe el planteamiento de una línea de investigación sobre soluciones constructivas de la fachada ventilada de piedra que resuelva requerimientos formales y técnicos desde los procesos propios de la creación arquitectónica.

\subsection{La fachada de piedra}

La tectónica de la piedra - de los muros portantes de una hoja- se perdió en aras de soluciones más eficientes y económicas, pero que permiten mantener la percepción de la piedra, de su textura, color o labra. Alcanzamos así las soluciones de anclajes en vuelo con hoja de piedra independiente ${ }^{3}$, pasando de los revestimientos calizos de las pirámides a las soluciones de aplacados de la antigüedad o a la citada solución de anclaje visto de Otto Wagner en la Karlplatz, sin olvidar el delicado muro de ónice del Pabellón Barcelona de Mies van der Rohe, todos ellos amorterados y/o grapados. Esta solución ha sido superada técnicamente por la difusión del rain-screen anglosajón, que busca la estanqueidad en una cámara de aire ventilada, sellada o nó ${ }^{4}$.

Un posible antecedente técnico del rain-screen lo encontramos en la solución utilizada por los romanos en sus construcciones de los hipocaustos (Fig. 02). Esta solución constructiva, de la cual están expuestos algunos restos arqueológicos en el British Museum de Londres, se atribuye al ingeniero romano del siglo pri- 


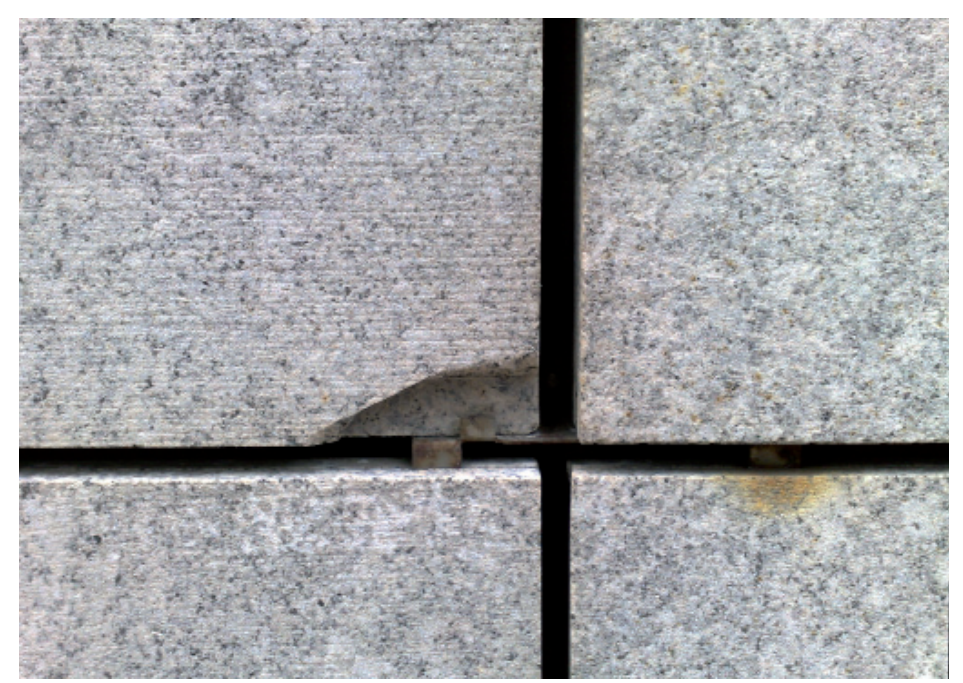

3 Lesión en una fachada ventilada de piedra en A Coruña; rotura en la acanaladura de la placa de granito sustentada con uñas de acero inoxidable. Se aprecian manchas de óxido del anclaje por la cercanía del mar.

mero Cayo Sergio Orata 5 . Pese a este antecedente, la solución ha sido una propuesta intuitiva, apoyada en el juicio por el uso, lejos de los métodos de investigación científica y de creación de sistemas ${ }^{6}$.

El primer estudio técnico sobre el rain-screen (fachada ventilada) lo encontramos en 1946 en un texto de Johansson, comentado posteriormente por Neil Hutcheon en 1953 como un sistema idealizado pero resoluble técnicamente, que cuenta con el acierto de utilizar materiales con un rango acotado de requerimientos? ${ }^{7}$. Es de destacar la publicación del ańo 1963 donde G.K. Garden analiza los procesos de penetración del agua en una fachada, introduciendo el concepto de pantalla de lluvia abierta para enunciar el funcionamiento de la fachada ventilada ${ }^{8}$.

\subsection{La fachada de piedra trasventilada anclada mecánicamente}

La fachada trasventilada mejoró la estanqueidad, pero concentró la complejidad técnica en el anclaje de la piedra. Y, una vez más, los aspectos formales exigidos por los arquitectos decantaron la solución hacia los anclajes ocultos, con los siguientes problemas:

- Concentración de tensiones en la placa.

- Independencia entre el número de anclajes y el tamaño, peso o espesor de la piedra.

- Manipulación de la placa pétrea con perforaciones afectadas por procesos de hielo-deshielo de la posible agua acumulada en ellos.
- Corrosión de los anclajes, o aparición de pares galvánicos con la subestructura.

- Regulación precisa y compleja, con abundante estructura auxiliar.

- Tensiones no previstas por montaje defectuoso (encaje forzado del anclaje).

- Modificación de la solución en zócalos por posibles impactos.

- Cada piedra debe de caracterizarse para el anclaje mecánico.

- Dificultad de mantenimiento o sustitución de cualquier pieza (Fig. 03).

\section{ANTECEDENTES}

En el año 2000 se plantea en nuestro estudio profesional la posibilidad de construir una fachada para una vivienda unifamiliar con piezas de pizarra de pequeño formato. La geometría del proyecto se resolvía con dos bloques pétreos unidos por un tercer volumen ligero de madera, que resolvía los accesos y las comunicaciones. La solución constructiva del cerramiento de madera se realizaba mediante un pegado elástico: el sistema Sikatack Panel registrado por Sika. La dificultad técnica del anclaje mecánico de la pizarra, su multiplicidad y la consecuente inviabilidad económica, nos obligaron a fijarnos en el sistema de sujeción de los paneles de madera, como una propuesta técnica 

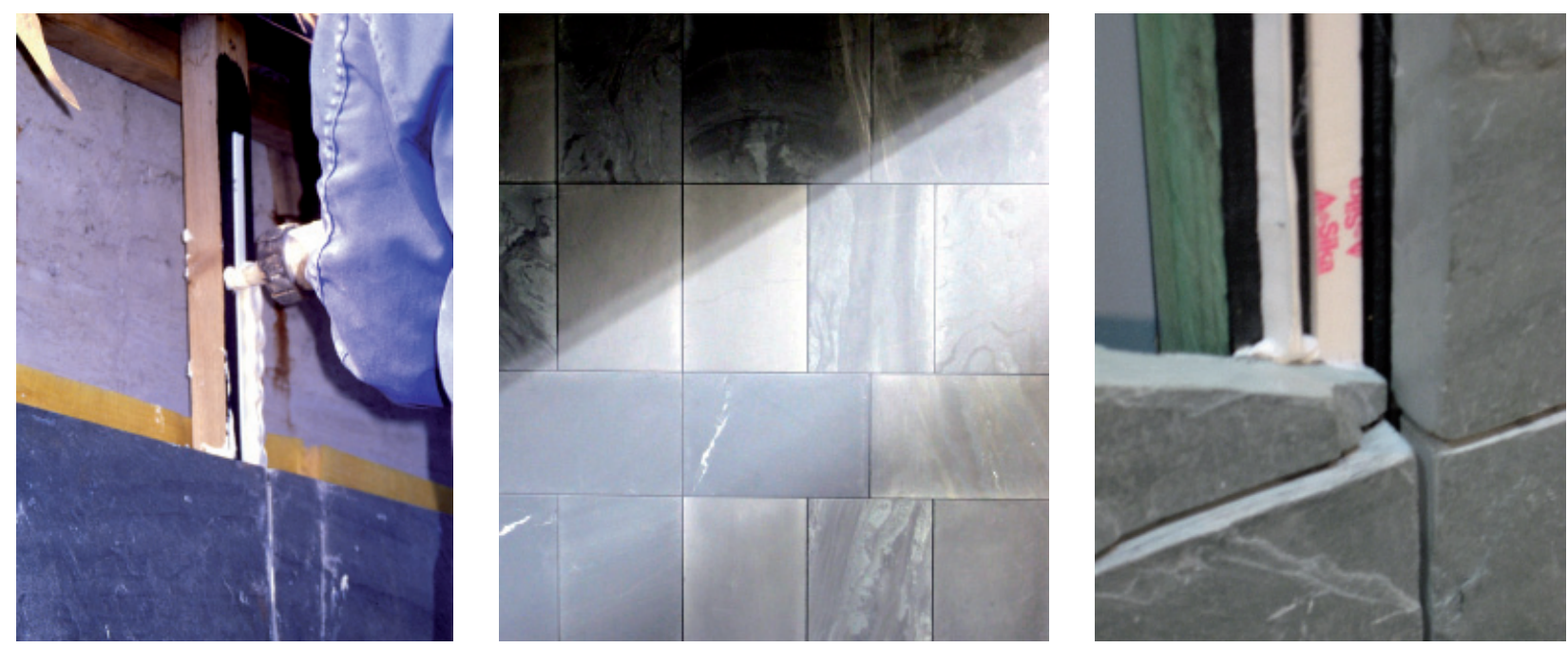

4 (Izda.) QRC SC arquitectos, Vivienda unifamiliar, Cambre (A Coruña, 2000); primera aplicación real donde se aprecia la singularidad de la ejecución. 5 (Centro) QRC SC arquitectos, Centro de Salud, Ortigueira (A Coruña, 2003); detalle de pizarra pulida con diferentes tamaños de piezas sustentadas mediante anclajes elásticos.

6 (Dcha.) Sika SA; detalle de laboratorio de pegado elástico de placas de pizarras, donde se aprecia el rastrel imprimado de madera tratada, las cintas de doble cara de pegado inicial y el cordón de masilla.

innovadora para el aplacado de piedra, aunque el sistema Sikatack Panel era para paneles ligeros y no para piedra. Tras unos pequeños ensayos caseros positivos y con la inestimable colaboración de la propiedad" nos lanzamos a su ejecución a finales del ańo 2001, usando otras masillas de la marca Sika y constatando que ha perdurado perfectamente hasta el día de hoy (Fig. 04).

Animados por esta realización, nos planteamos en al año 2001 la reutilización de los desechos de la industria pizarrera en una fachada para un Centro de Salud en Ortigueira (A Coruña). Las piezas partidas, de espesores y formato dispar, se nos antojaban que aportaban una expresividad necesaria a los volúmenes de este proyecto. Pero la solución de anclaje mecánico de la pizarra se presentaba imposible con las técnicas existentes, ante la variedad de formatos, dimensiones y la facilidad de exfoliación natural de la pizarra ante la perforación. Para adoptar la solución de pegado elástico de aplacado pétreo tuvimos que sistematizar el proceso y prever los ensayos de control de calidad para conocer el comportamiento a largo plazo. En el año 2003, el laboratorio de la empresa Control y Estudios realizó los ensayos de caracterización (tracción, cortante y envejecimiento) con resultado positivo para esta obra. Finalmente, la solución se limitó a una fachada de un patio interior, pese a la expresa contraindicación de Sika, por no tratarse de un sistema ligero (Fig. 05).

La solución de aplacados pétreos con pegado flexible se presentó en los "III Encontros de Arquitectura en
Santiago de Compostela" (Fig. 06) en octubre del año 2001, y se construyeron diferentes ejemplos siguiendo el sistema (Fig. 07). Con posterioridad, tras una serie de ensayos de pelado realizados en el 2005 sobre adhesión sobre pizarras, la empresa de elaborados químicos para la construcción Sika presentó un catálogo de soluciones de fachada ventilada con pegado elástico, donde se muestran las obras realizadas por el estudio con anterioridad.

\section{DESCRIPCIÓN DEL SISTEMA. PEGADO ELÁSTICO DE PIEDRA EN FACHADAS TRANSVENTILADAS}

La solución propuesta solventa la libertad de composición demandada (Fig. 08). Realiza una unión elástica, estableciendo una relación de proporcionalidad entre el milímetro cuadrado de adhesivo elástico y el metro cuadrado de fachada-peso-succión, sin que el tamaño de la pieza repercuta en el coste de la sujeción, sin imponer juntas coincidentes o contrapeadas, sin determinar geometricamente las piezas de aplacado, sin manipulaciones de la placa de piedra, sin provocar corrosión ni pares galvánicos, y con posibilidad de reponer cada pieza (mediante corte con hilo de acero del cordón adhesivo) manteniendo el sistema constructivo. Además, aporta atenuación acústica, absorción de movimientos, absorción de imperfecciones constructivas y economía de costes, tanto de construcción como de mantenimiento, aunque sigue necesitando de caracterización de la piedra para el anclaje elástico, al igual que el mecánico. 

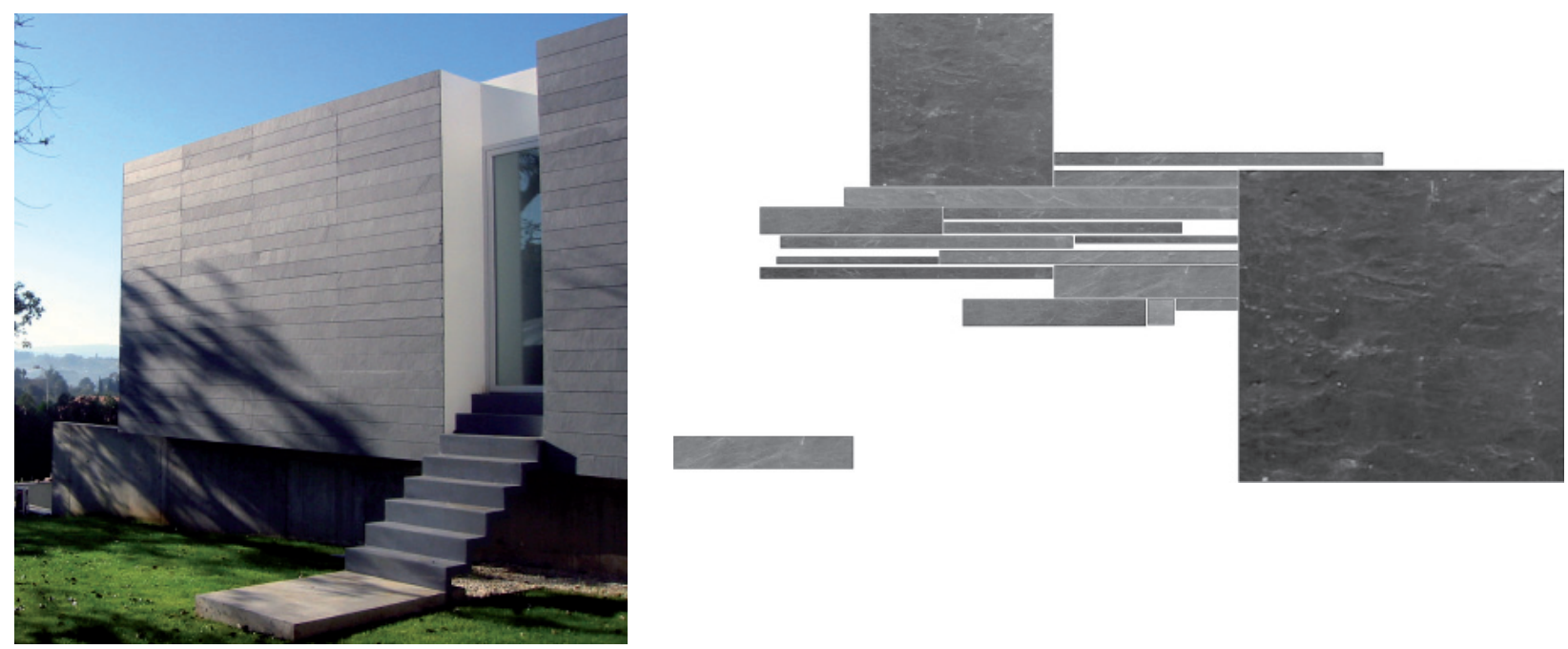

7 (Izda.) Alfonso Fernández Bernárdez, Vivienda unifamiliar, Oleiros (A Coruña, 2002/05); fachada ventilada de pizarra verde con sistema de pegado elástico.

8 (Dcha.) Antonio Raya de Blas, dibujo de composición teórica; se constata la libertad de criterio de colocación, donde el único requerimiento es la superficie de cordón de pegado elástico y que toda pieza esté apoyada en dos líneas.

\subsection{Elementos del sistema}

El sistema está integrado por los siguientes elementos:

Subestructura de apoyo, compuesta por montantes y/o travesaños anclados a la hoja interior o a los forjados. Esta subestructura se ejecutará en aluminio, madera tratada, acero inoxidable, piezas de hormigón o plásticos. La imprimación para el pegado se definirá en función del material seleccionado (Fig. 09).

Imprimación, que mejore la adherencia entre la masilla de pegado elástico, la subestructura y la pieza. Ésta dependerá del material.

Cinta de doble cara adhesiva para la fijación inicial de las piezas de piedra mientras la masilla polimeriza, garantizando el espesor mínimo del cordón de pegado. La cinta deberá ser elástica, y debe poder adaptarse a las posibles irregularidades de la pieza (por ejemplo, pizarra).

Masilla elástica adhesiva, de base poliuretano monocomponente o cualquier otra con suficiente capacidad adherente en el tiempo.

\subsection{Requerimientos del sistema.}

\section{Criterios de diseńo y construcción}

- Toda placa irá apoyada en —al menos— dos cordones continuos separados entre sí.
- La separación entre montantes o travesaños viene condicionada por el requerimiento anterior y por la superficie de pegado, calculada para resistir las acciones sobre la placa.

- La posición de los rastreles no obliga a una coincidencia con la junta de aplacado.

- Las dimensiones de cálculo del cordón son 10 $\mathrm{mm}$. de ancho y $3 \mathrm{~mm}$. de espesor.

- Los huecos, triedros, albardillas o remates singulares no requieren de soluciones especiales.

- Las superficies de pegado deben estar secas y protegidas de la lluvia durante las primeras horas de polimerización.

- La subestructura debe de garantizar la transmisión de los esfuerzos de la fachada a la estructura principal.

- Se atenderán las especificaciones del fabricante de la masilla.

\section{ENSAYOS}

En el transcurso de la ejecución de la obra del Centro de Salud de Ortigueira en el año 2003, como dirección de obra, encargamos a la empresa Control y Estudios (empresa adjudicataria del control de calidad) una serie de ensayos de la solución descrita ajustada a esta obra. Concretamente, se ensaya la utilización de pizarra sobre rastreles de madera de pino tratada, 


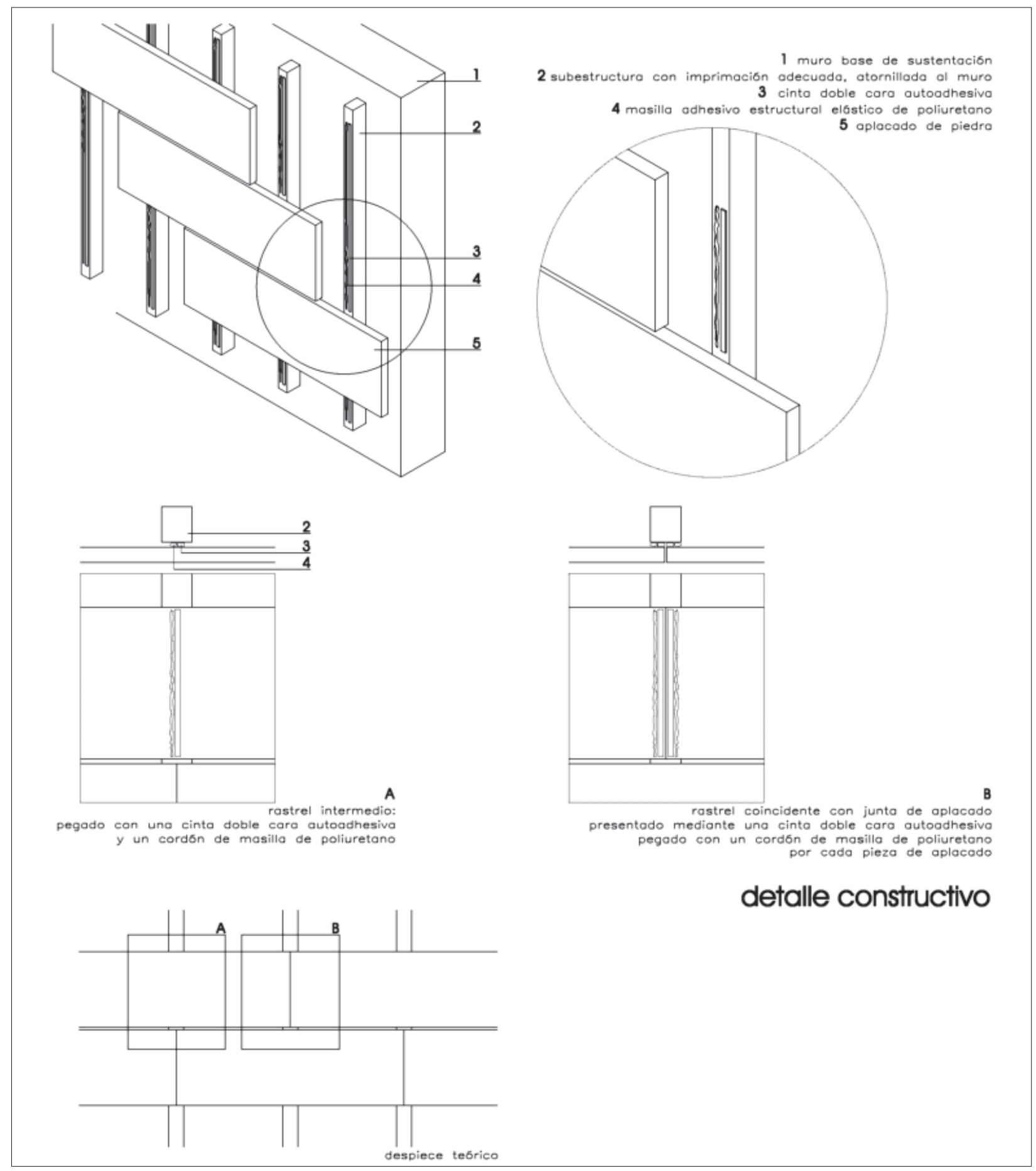

9 Antonio Raya de Blas, detalle constructivo del sistema de pegado elástico de fachada trasventilada de piedra.

usando una imprimación y masilla de poliuretano. Los ensayos son:

Ensayos de caracterización de la unión adhesiva con masilla de poliuretano Sikabond T2 e imprimación SikaTack Panel Primer.

- Peso propio. Resistencia inicial a cortante y envejecimiento.
- Succión de viento. Resistencia inicial a tracción y envejecimiento.

Ensayos de comparación

- Ensayo a tracción de tres probetas de $350 \times 300$ x $20 \mathrm{~mm}$. pegadas elásticamente, con anclaje mecánico mediante un perfil continuo de aluminio y uñas de acero inoxidable. 


\begin{tabular}{|c|c|c|c|c|c|c|}
\hline \multicolumn{7}{|c|}{$\begin{array}{c}\text { ENSAYO A CORTANTE PEGADO ELÁSTICO } \\
\text { Muestra: } 100 \times 25 \times 5 \text { mm. Solape: } 15 \text { mm. Espesor de pegado: } 4 \text { mm. } \\
\text { Velocidad: } 10 \text { mm./min. Clase escala máquina: 0,5 }\end{array}$} \\
\hline & & $\mathrm{Fm}$ & Sm & DI en rotura & Ancho & Largo \\
\hline & & $(\mathrm{N})$ & (N/mm2) & $(\mathrm{mm})$ & $(\mathrm{mm})$ & $(\mathrm{mm})$ \\
\hline \multirow{2}{*}{ inicial* } & media & 186,5 & 1,037 & 13,10 & 12,17 & 15,03 \\
\hline & desviación est & 16,9 & 0,199 & 1,27 & 1,01 & 1,59 \\
\hline \multirow{2}{*}{$\begin{array}{l}\text { inmersión** } \\
60^{\circ} \text { C } 30 \text { días }\end{array}$} & media & 153,8 & 0,769 & 6,78 & 13,34 & 15,01 \\
\hline & desviación est & 83,1 & 0,428 & 3,00 & 0,11 & 0,70 \\
\hline \multirow{2}{*}{$\begin{array}{l}\text { inmersión } \\
165 \text { días }\end{array}$} & media & 165.7 & 0,803 & 6,801 & 13,68 & 15,16 \\
\hline & desviación est & 40,9 & 0,225 & 1,392 & 0,40 & 0,40 \\
\hline \multicolumn{7}{|c|}{ 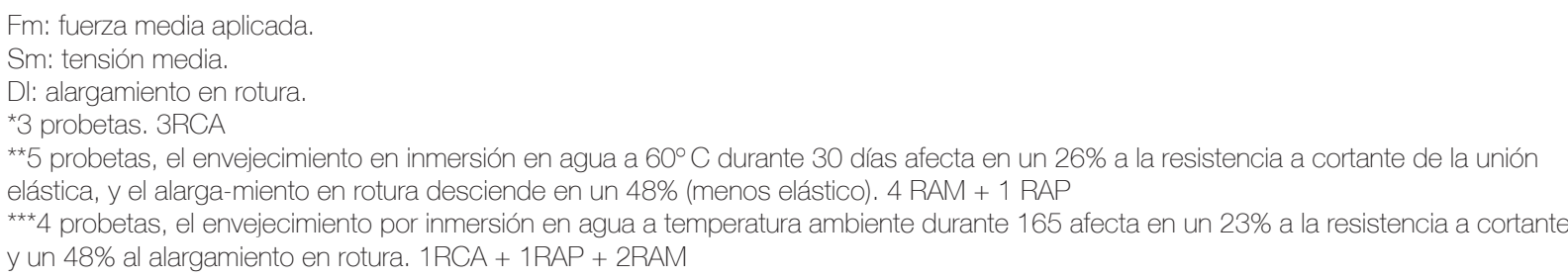 } \\
\hline
\end{tabular}

Los ensayos con probetas de dimensiones similares a las puestas en obra con diferentes sistemas de anclaje nos permitieron establecer una primera valoración del comportamiento del pegado elástico por comparación. En las tablas anexas se resumen los valores obtenidos y se indica el tipo de rotura ${ }^{10}$ :

Rotura cohesiva:

- RCA en el adhesivo.

- RCM en la madera.

- RCP en la pizarra.

Rotura adhesiva:

- $\quad$ RAM en la interfaz con la madera.

- RAP en la interfaz con la pizarra.

Los ensayos se han realizado para una obra concreta con solución de madera y pizarra, ambos materiales muy afectados por el envejecimiento acelerado. En cualquier caso, es necesario caracterizar el anclaje según UNE-22203 4.1.3 b), considerado como ensayo tecnológico.

Los ensayos realizados deberían llegar hasta los 20 para poder determinar la resistencia de forma estadística. Por tanto, es necesario proceder a la realización de los ensayos de caracterización para cada ejecución en concreto; lo que, por otra parte, parece totalmente recomendable, atendiendo a las variaciones que la piedra natural puede presentar.
El adhesivo disminuye sus propiedades mecánicas de forma importante en el ensayo de envejecimiento por inmersión a $60^{\circ} \mathrm{C}$ durante 30 días: un $26 \%$ a cortante y un $16 \%$ en tracción. La carga de succión de viento es la más desfavorable (DB-SE-AE-3.3.1) y dependerá de diversos criterios: posición relativa en la fachada (centro, esquina), espesor de la cámara y su compartimentación para ecualizado de presiones ${ }^{11}$. Para el edificio estudiado ( $6 \mathrm{~m}$. de altura al borde del mar) y aplicando el actual CTE, la succión máxima es de:

$$
q_{e}=a_{b} \times C_{e} \times C_{p}=50 \times 2,7 \times-0,4=-54 \mathrm{Kg} / \mathrm{m}^{2}
$$

Adoptando como tensión de cálculo la mitad de la de rotura, $0,864 \mathrm{~N} / \mathrm{mm}^{2}\left(8,64 / 2=4,3 \mathrm{Kg} / \mathrm{cm}^{2}\right)$ y adoptando como coeficiente de seguridad 4,5, sería necesaria una superficie adherida de $56,26 \mathrm{~cm}^{2}$ por metro cuadrado de fachada ${ }^{12}$. En los ensayos realizados sobre probetas de $350 \times 300 \times 20 \mathrm{~mm}$., el ancho del cordón adhesivo era de $2 \mathrm{~cm}$.; adoptando como ancho de cálculo la mitad, la longitud de pegado sería como mínimo de $57 \mathrm{~cm} / \mathrm{m}^{2}$, siendo mucho más restrictiva la condición geométrica de apoyo. Si en vez de la carga prevista para el edificio adoptamos $210 \mathrm{Kg} /$ $\mathrm{m}^{2}$ (la utilizada por el Instituto Eduardo Torroja en el DIT-353R) ${ }^{13}$ y repetimos el cálculo, sería necesario un cordón de $220 \mathrm{~cm}$. de longitud por cada metro cuadrado de fachada, prácticamente un rastrel de apoyo cada metro con su correspondiente cordón de pegado. Por otra parte, si la fachada estuviese bien sectorizada, la presión se ecualizaría y no existirían cargas signifi- 


\begin{tabular}{|c|c|c|c|c|c|c|}
\hline \multicolumn{7}{|c|}{$\begin{array}{l}\text { ENSAYO A TRACCIÓN PEGADO ELÁSTICO } \\
\text { Muestra: } 25 \times 25 \times 10 \text { mm. Espesor del cordón: } 2 \text { mm. } \\
\text { Velocidad } 5 \text { mm./min. Clase escala maquina: 0,5 }\end{array}$} \\
\hline & & Fm & $\mathrm{Sm}$ & DI en rotura & Ancho & Largo \\
\hline & & $(\mathrm{N})$ & (N/mm2) & $(\mathrm{mm})$ & $(\mathrm{mm})$ & $(\mathrm{mm})$ \\
\hline \multirow{2}{*}{ inicial* } & media & 607,0 & 1,027 & 4,523 & 23,76 & 24,71 \\
\hline & desviación est & 111.4 & 0,142 & 2,421 & 0,99 & 0,46 \\
\hline \multirow{2}{*}{$\begin{array}{l}\text { inmersión } n^{\star *} \\
60^{\circ} \text { C } 30 \text { días }\end{array}$} & media & 533,1 & 0,864 & 1,406 & 25,34 & 24,30 \\
\hline & desviación est & 240,6 & 0,386 & 0,966 & 0,55 & 0,71 \\
\hline \multirow{2}{*}{$\begin{array}{l}\text { inmersión }{ }^{\star * \star} \\
165 \text { días }\end{array}$} & media & 637,1 & 1,096 & 1,660 & 24,10 & 24,16 \\
\hline & desviación est & 96,4 & 0,166 & 0,423 & 0,89 & 1,14 \\
\hline \multicolumn{7}{|c|}{$\begin{array}{l}\text { Fm: fuerza media aplicada. } \\
\text { Sm: tensión media. } \\
\text { Dl: alargamiento en rotura. } \\
{ }^{* 5} \text { probetas. } 4 \text { RCA + } 1 \text { RCP } \\
\star \star 5 \text { probetas, el envejecimiento en inmersión en agua a } 60^{\circ} \mathrm{C} \text { durante } 30 \text { días afecta en un } 16 \% \text { a la resistencia a tracción de la unión } \\
\text { elástica, y el alarga-miento en rotura desciende en un } 69 \% \text {, aunque no resulta significativo por la rotura de la pizarra o del rastrel de apo- } \\
\text { yo. } 2 \text { roturas adhesiva con el rastrel de madera, } 1 \text { rotura adhesiva con la pizarra, } 1 \text { rotura cohesiva en la madera y } 1 \text { rotura cohesiva en } \\
\text { la pizarra. } \\
\star \star \star 5 \text { probetas, la resistencia tracción no se ve afectada y disminuye en un } 63 \% \text { el alargamiento en rotura. 3RAM + 1RCP + 1RCA }\end{array}$} \\
\hline
\end{tabular}

\begin{tabular}{|c|c|c|c|c|c|c|}
\hline \multicolumn{7}{|c|}{$\begin{array}{c}\text { ENSAYO COMPARATIVO DE SISTEMAS DE ANCLAJE } \\
\text { Muestra: } 350 \times 300 \times 20 \mathrm{~mm} \text {. } \\
\text { Velocidad: } 0,4 \mathrm{~mm} . / \mathrm{s} \text {. Clase escala maquina: } 1\end{array}$} \\
\hline & & $\mathrm{Fm}$ & $\mathrm{Sm}$ & DI en rotura & Ancho & Largo \\
\hline & & $(\mathrm{N})$ & $(\mathrm{N} / \mathrm{mm} 2)$ & $(\mathrm{mm})$ & $(\mathrm{mm})$ & $(\mathrm{mm})$ \\
\hline $\begin{array}{l}\text { pegado } \\
\text { elástico* }\end{array}$ & tracción & 5270 & 1,146 & - & 20 & 230 \\
\hline \multirow{2}{*}{$\begin{array}{l}\text { mecánico perfil } \\
\text { continuo de } \\
\text { Al** }^{\star \star}\end{array}$} & tracción & 5740 & - & - & - & - \\
\hline & cortante & & - & - & - & - \\
\hline \multirow{2}{*}{$\begin{array}{l}\text { mecánico } \\
\text { uñas de acero } \\
\text { inoxidable }\end{array}$} & tracción & 7535 & & & & \\
\hline & cortante & 8134 & & & & \\
\hline \multicolumn{7}{|c|}{$\begin{array}{l}\text { Fm: fuerza media aplicada. } \\
\text { Sm: tensión media. } \\
\text { Dl: alargamiento en rotura. } \\
\text { *La primera probeta rompe por deslajamiento de la pizarra en la unión epoxídica del pistón para una carga de } 830 \text { N. La segunda rotur } \\
\text { es cohesiva en el adhesivo a } 5.270 \mathrm{~N} \text {. } \\
\text { **Fallo por deformación de los perfiles. } \\
\text { ***Fallo por deformación de los ganchos. }\end{array}$} \\
\hline
\end{tabular}

cativas de viento sobre la hoja de piedra; pero hemos dimensionado el caso más desfavorable sin ecualización de cámara.

De igual forma se pude proceder para la comprobación a peso propio, en nuestro caso $36 \mathrm{Kg} / \mathrm{m}^{2}$. Con un coeficiente de seguridad 4,5 y una tensión de cálculo a cortante de $3,84 \mathrm{Kg} / \mathrm{cm}^{2}$, la resistencia del cordón de
$57 \mathrm{~cm}$. sería de $218 \mathrm{Kg} / \mathrm{m}^{2}$; y para el cordón de 220 $\mathrm{cm}$., la resistencia alcanzaría los $844 \mathrm{Kg} / \mathrm{m}^{2}$, siempre muy por encima de lo demandado incluso con aplacados de mayor espesor.

El ensayo testigo de placas de pizarra de 350x300×20 mm, con pegado elástico y con anclaje mecánico, da resultados insuficientes, pero dentro una banda equiparable. 
El sistema, con todas las ventajas indicadas, es capaz de asumir los requerimientos técnicos a los que está sometido, aunque en el caso de usar piedra natural debe constatarse en cada obra.

\section{CONCLUSIONES}

- La solución es perfectamente valida, aunque atendiendo a la UNE-22203 4.1.3, es necesario realizar ensayos de caracterización para su extrapolación a otra obra, como ocurre con los anclajes mecánicos. Lo que nos permite plantear este sistema como solución técnica en fachadas ventiladas, aunque necesita de un mayor desarrollo.

- El sistema soluciona gran parte de los problemas descritos para un anclaje mecánico: libertad de composición, proporcionalidad anclaje-peso-acciones, independencia anclaje-superficie de la pieza, no-imposición de juntas coincidentes o contrapeadas, indeterminismo geométrico de las piezas, ausencia de manipulaciones de la placa de piedra, ausencia de corrosión o pares galvánicos, y posibilidad de reponer cada pieza independientemente con el mismo sistema.
- La temperatura es uno de los factores que más afecta a los adhesivos químicos en su comportamiento; por tanto, debe ser evaluada muy detenidamente.

- La inmersión en agua durante largos periodos no afecta al comportamiento de la masilla de poliuretano ensayada.

- Mediante ensayos de identificación, el sistema se puede aplicar a otras subestructuras (aluminio, acero inoxidable) más habituales, y con ensayos tecnológicos a otras piedras (granito, mármol) o cerámicas, entre otros.

- Al existir una construcción real con más de diez años y otras con diferentes edades se puede seguir y ensayar su comportamiento real fuera de laboratorio.

- Finalmente, se están evaluando sistemas alternativos de sujeción provisional a la cinta de doble cara para solucionar la sustentación de la placa durante el proceso de secado de la masilla.

\section{Notas}

1. Cf. Jaume Avellaneda Díaz-Grande e Ignacio Paricio Ansuategui, Los revestimientos de piedra (Barcelona: Bisagra, 1999), 6.

2. Cf. Thomas Kuhn, La estructura de las revoluciones cientificas (Madrid: Fondo de Cultura Económica de España, 2005). Enuncia las tres etapas del pensamiento científico: precientífica, ciencia normal y ciencia revolucionaria.

3. Cf. J.M. Anderson y J.R. Gill, Rainscreen Cladding: A Guide to Design Principles and Practice (London: Butterworth-Heinemann, 1988), 3. Establece el antecedente en los graneros noruegos para la fachada trasventilada.

4. Cf. Cristina Pardal March e Ignacio Paricio Ansuategui, La fachada ventilada y ligera (Barcelona: Bisagra, 2006), 14. Existen referencias en Espańa como el tabique pluvial catalán o diversas soluciones gallegas.

5. Cf. Plinio (Hist. Nat., IX, 168) las llama balneae pensiles, literalmente baños colgantes, aunque la traducción más exacta puede ser piscinas calientes.

6. Cf. G.O. Handegord, "The Performance of Exterior Walls" (Prefacio presentado en Building Science Insight 1982-Exterior walls: understanding the problems, Ottawa, 1982), 5-14

7. Cf. C-H. Johansson, "The influence of moisture on the heat conductance for bricks", Byggmastaren 7 (1946): 117-124. Describe la pantalla de protección contra la lluvia, con aislamiento exterior, ventilada y traspirable; N.B. Hutcheon, "Fundamental Considerations in the Design of Exterior Walls for Buildings". National Research Council NRC 3057 (1953): 25.

8. Cf. George Kirby Garden, "Rain penetration and its control”, Canadian Building Digests 40 (1963): 1-4

9. Saúl González Casas, propietario y arquitecto técnico de la dirección facultativa.

10. Cf. EN-12004-2008, Adhesivos para baldosas cerámicas. Requisitos, evaluación de la conformidad y designación (Madrid: AENOR 2008), 16. Clasificado el fallo según el tipo de rotura: rotura adhesiva, cuando se produce en la interfaz entre el adhesivo y los elementos unidos; y rotura cohesiva, cuando se produce en el seno del adhesivo.

11. Cf. Joaquín Fernández Madrid, "Study of influence of wind pressure and rain conditions in rain screen walls", Structural Survey 28/1 (2010): 51. En este artículo se concluye la influencia del ancho y compartimentación de las cámaras ventiladas en las presiones dinámicas por viento en cada hoja (exterior e interior).

12. Cf. Eduardo Lahoz Ruiz, "Caracterización de la resistencia de una fijación oculta mecánico adhesiva para aplacados de piedra natural en un sistema de fachada ventilada” (Actas de las II Jornadas de Investigación en Construcción), Madrid: IETCC-CSIC, 2008). Cita la norma UNI32045130 que recomienda un coeficiente de seguridad para aplacados entre 4,5 y 9.

13. Cf. Instituto Eduardo Torroja CSIC, "DIT-353 Sistema de revestimiento de fachadas Mecanofas Karrat S-7", (Madrid: IETCC-CSIC, 2007 ), 8. Antecedente de pegado elástico con encastre por acanaladura para sujeción mecánica al perfil continuo de cuelgue de la estructura auxiliar. DIT Renovado en el año 2007 sobre ensayos realizados en 1999 y 2000. 


\section{Referencias}

Anderson, J.M. y J.R. Gill. Rainscreen Cladding: A Guide to Design Principles and Practice. London: Butterworth-Heinemann, 1988.

Avellaneda Díaz-Grande, Jaume e Ignacio Paricio Ansuategui. Los revestimientos de piedra. Barcelona: Bisagra, 1999.

Fernández Madrid, Joaquín. "Study of influence of wind pressure and rain conditions in rain screen walls". Structural Survey 28/1 (2010): 46-52.

Garden, George Kirby. "Rain penetration and its control". Canadian Building Digests 40 (1963): 1-4.

Handegord, G.O. "The Performance of Exterior Walls". Prefacio presentado en Building Science Insight 1982-Exterior walls: understanding the problems. Ottawa: National Research Council Canada, 1982.

Hutcheon, N.B. "Fundamental Considerations in the Design of Exterior Walls for Buildings". National Research Council 3057 (1953): 1-25.

Instituto Eduardo Torroja-CSIC. DIT-353 Sistema de revestimiento de fachadas Mecanofas Karrat S-7. Madrid: IETCC-CSIC, 2007.

Johansson, C.H. "The influence of moisture on the heat conductance for bricks". Byggmastaren 7 (1946): 117-124.

Kuhn, Thomas. La estructura de las revoluciones cientificas. Madrid: Fondo de Cultura Económica de Espańa, 2005.

Lahoz Ruiz, Eduardo. "Caracterización de la resistencia de una fijación oculta mecánico adhesiva para aplacados de piedra natural en un sistema de fachada ventilada”. Actas de las II Jornadas de Investigación en Construcción. Madrid: IETCC-CSIC, 2008.

Money, J.H. "Garden Hill, Sussex: Interim Report". Britannia 8 (1977): 339-50.

Pardal March, Cristina e Ignacio Paricio Ansuategui. La fachada ventilada y ligera. Barcelona: Bisagra, 2006.

\section{Procedencia de las ilustraciones}

Fig. 01. Chogenbo.

Fig. 02-06 y 08-09. Antonio Raya de Blas.

Fig. 07. Héctor Fernández Santos-Díez.

\section{Sobre los autores}

Antonio Raya de Blas, Santiago Sánchez Iglesias y Enrique Antelo Tudela son arquitectos y profesores del Departamento de Construcciones Arquitectónicas de la ETSA de la Universidade da Coruña; Cristóbal Crespo González, arquitecto, es profesor del Departamento de Proyectos Arquitectónicos y Urbanismo del mismo centro. En 2002 crean Vier arquitectos (A Coruña), en continuidad con QRC arquitectos. El despacho dirige su atención principalmente hacia programas institucionales y residenciales, experimentando con nuevas técnicas y materiales.

adeblas@udc.es 